\title{
Summary functions in the analysis of spatial point patterns
}

\author{
B.T. SCOTT
}

This thesis presents a detailed comparison of methods for the statistical testing of spatial point patterns. In particular, methods based on summary functions of spatial point patterns are considered. The basis of this comparison is a series of large-scale simulation studies, used to estimate the power of various statistical tests for detecting deviations from the Poisson process. Under investigation is the approach of converting two summary functions into a single test statistic quantifying the discrepancy between two point processes.

This approach is explored by considering the recent development of a series of summary functions, defined by the Minkowski functionals of a point process dilation. These summary functions present a method for generalisation of the empty space function. As such they provide the opportunity to compare the discriminating ability of various summary functions derived from the same measurement of a point pattern; in this case the distance from an arbitrary point in space to nearest point of the process. Of particular interest is the observation that the probability density (or derivative) of the empty space function appears to be more sensitive than the empty space function itself.

Methods for constructing test statistics from summary functions are considered in detail, with application to these Minkowski summary functions and other common summary functions. This work highlights some of the important considerations when constructing spatial goodness-of-fit tests from summary functions. Special attention is given to interactions between the choice of summary function and discrepancy measure, highlighting the complex nature of these interactions. A non-parametric approach to measuring the discrepancy between two summary functions is then devised.

Further to this, a fully non-parametric approach to goodness-of-fit testing is developed. This method, based directly on the measurements taken from the point pattern, removes the need to consider summary functions. Initially developed as a univariate goodness-of-fit technique, this method is also applied to spatial nearest neighbour distances with promising results. Included as part of this work is a review of existing

Received 9th January, 2002.

Thesis submitted to The University of Western Australia, March 2001. Degree approved, December 2001. Supervisors: Professor A. Baddeley and Dr N. Campbell, CSIRO Mathematical and Information Sciences.

Copyright Clearance Centre, Inc. Serial-fee code: 0004-9727/02 \$A2.00+0.00. 
techniques, including a number of methods that are shown to be equivalent to this 'new' method.

Implicit in the estimation of summary functions within bounded observation windows is the problem of edge effects: the censoring of measurements dictated by the existence of a restricted observation region, beyond which the point pattern is unobserved. Although not essential when constructing a Monte Carlo goodness-of-fit test, some form of edge correction is generally utilised in order to account for these edge effects, especially when the summary function is to be compared directly with the theoretical summary function of some point process.

Two novel approaches to the treatment of edge effects are presented. The first of these, the result of joint work, relates to the impact of ignoring the need for edge corrections when estimating van Lieshout and Baddeley's $J$ function from a Poisson process realisation. In particular, it is shown that the uncorrected $J$ function estimator is ratio-unbiased for the Poisson process. Consequently the uncorrected $J$ function estimator can be used in the qualitative assessment of complete spatial randomness. Interestingly, this approach is found to produce superior results in many cases.

The second method developed for handling edge effects is somewhat more conventional, in that it is a form of edge correction, applicable to estimating the nearest neighbour distance distribution. Based on a hazard function estimate, following the derivation of the Kaplan-Meier estimator of survival analysis, this estimator appears to have superior efficiency compared with conventional edge corrections.

The thesis concludes with a discussion of the significance of this body of work to the field of testing spatial point patterns, highlighting a number of areas with the potential for further work.

Defence Systems Analysis Division

DSTO, Fishermans Bend

GPO Box 4331

Melbourne Vic. 3001

Australia

e-mail: Bryan.Scott@dsto.defence.gov.au 